\title{
THE DeClaration OF HELSINKI REVISED BY THE WORLD MEDICAL ORGANISATION, EDINBURGH 2000
}

\author{
Erwin Deutsch*
}

This is an edited version of a paper delivered at a conference in Gijon, Spain in 2001. Professor Deutsch provides an introduction to and overview of the recently revised Declaration of Helsinki.

\section{HISTORY}

The Declaration of Helsinki was promulgated first in 1962. In 1975 it was totally rewritten and thus both the Ten Principles of Nuremberg and the Declaration of Helsinki of 1962 were replaced. These international rules put the benefit and the well-being of the experimental subject in first place and insist on weighing the benefit and the danger of the experiment against each other. It is necessary to obtain informed consent which can be given for incapacitated persons by their legal representatives. If there is experimentation by doctors with their own patients special care has to be taken in order that there is no undue influence on the patient.

In 1975 there were two big changes in the set-up and the working of research protocols. The first big change was the establishment of so-called ethical committees. They are not named ethical committees, but especially appointed in depen dent committees for consideration, comment and guidance. A later version of the Declaration of Helsinki required that the committee work according to the laws and regulations of the country in which the research takes place.

The second change concerned the publication of research papers. The research protocol should contain a statement of the ethical considerations involved. Research papers deemed

\footnotetext{
* Professor of Law, University of Göttingen.
} 
unethical should not be published. The editors and publishers of the major scientific journals interpreted this rule as requiring the clearance by an ethical committee for the research protocol and providing the publishers with a copy of the opinion of the ethical committee. That way all research on human beings suddenly came before ethical committees. If the researchers wanted to publish their findings later on, they had to have the clearance by their ethical com mittee.

\section{THE DEBATE ABOUT REFORMING THE DECLARATION OF HELSINKI \\ A Additions to the Declaration of Helsinki}

The Declaration of Helsinki of 1975 has been amen ded three times: in Venice 1983, in Hong Kong 1989 and in Somerset West in 1996. In all instances there were minor additions, for instance about informed consent by minors or the legal recognition of ethical committees by local law.

\section{B The Plan by the American Medical Association}

In 1999 the American Medical Association tried to change the Declaration of Helsinki totally. Not only should the established rules be changed but the wording of the Declaration as well. The American Medical Association wanted the Declaration to adopt the following principles:

- there should be no differentiation between therapeutic experimentation and purely scientific experimentation;

- ethical committees would become review committees who had to look at the research protocol for scientific validity as well;

- $\quad$ ethical committees should not be local review committees but general ones;

- the Declaration of Helsinki should not bind just medical doctors but all other persons who are doing medical research on humans eg psychologists, nurses, physicists, chemists;

- the principle of informed consent and of acceptability were mixed up and looked at under different aspects;

- therapeutic and non-therapeutic trials were treated equally. 


\section{European Reaction}

The European countries especially Great Britain, France, and Germany disagreed with the American suggestion. They wanted especially to keep the differentiation between purely scientific research and therapeutical trials. They insisted on having the ethical committee at the place of experimentation giving its opinion. Finally, they preferred presumed consent by persons unable to give it verbally, in place of asking the ethical committee.

\section{Drafting Committee}

The World Medical Association gave the task of drafting the new Declaration of Helsinki to a committee of three members who were presidents of their respective medical associations. They met a few times and finally presented a draft to the 52 WMA General Assembly at Edinburgh that became the new Declaration of Helsinki 2000.

\section{THE NEW DECLARATION OF HELSINKI 2000'}

\section{A Introduction}

The new Declaration of Helsinki is divided in three parts. First there is an introduction consisting of nine rules. It is followed by eighteen basic principles for all medical research. Finally there are five additional principles for medical research combined with medical care. As far as the introduction is concerned, rule 4 states that medical progress is based on research which ultimately must rest in part on experimentation involving human subjects. It is followed by rule 5, which says that in medical research on human subjects consideration of the well-being of the human subject should take precedence over the interests of science and society. The same dual approach is apparent in the following rules. In rule 7 it is said that in current medical practice and in medical research, most prophylactic, diagnostic, and therapeutic procedures involve risks and burdens. Then rule 8 , which relates to special groups, says that medical research is subject to ethical standards that promote respect for all human beings and protect their health and rights. Some research populations are vulnerable and need special protection. The particular needs of the economically and medically disadvantaged must be recognised. Special attention is also required for those who cannot give or refuse consent for themselves, for those who may be subject to giving consent under duress, for those who will not benefit personally from the research and for those, for whom the

\footnotetext{
1 The text of the Declaration is available at $<$ http: // o nlineethics.org/reseth/helsinki.html $>$.
} 
research is combined with care. That rule relates to endangered groups who should be specially protected.

The final rule, 9, concerns international public law. First, research investigators are told to be aware of the ethical, legal, and regulatory requirements for research on human subjects in their own countries, as well as applicable international requirements. That means, all researchers should know the local and the international rules concerning medical research, be they statutes, ethical rules or rules more of a regulatory manner. Second, rule 9 concerns itself with the relation of the Declaration of Helsinki to the local law. No ethical, legal or regulatory requirement should be allowed to reduce or eliminate any of the protection for human subjects set forth in this Declaration. This rule is hard to explain. On the surface, it states that as far as the protection of human subjects is concerned, the Declaration of Helsinki takes precedence over local rules. There is no problem if the local law falls just short of the Declaration of Helsin ki, ie protecting the human subjects not as far as the Declaration does. The real problem arises when the national law requires some commission or omission that endangers the human subject or reduces its legal standing. Take for instance the case where a researcher is doing a controlled clinical study on persons without informing them. According to international public law, the national rules take precedence over the Declaration of Helsinki because they are the law of the land and the Declaration just states ethical principles. But if the rights of human subjects are totally abridged, especially if they constitute basic rights of the person, then the international instruments for the protection of the person will help, eg the European Convention on Hum an Rights.

\section{B Basic Principles for all Medical Research}

In quite a few of the basic principles the Declaration undertakes to set up rules for acceptable risk, informed consent and other requirements for research. Rule 10 states that it is a duty of the physician in medical research to protect the life, health, privacy, and dignity of the human subject. Before research starts, the researcher has to make sure that his research project conforms to generally accepted scientific principles, is based on a thorough knowledge of the scientific literature or other relevant sources of information and on adequate laboratory and, where appropriate, animal experimentation. As in the old Declaration of Helsinki, rule 12 provides that appropriate caution must be exercised in the conduct of research which may effect the environment, and the welfare of animals.

The protection is given not just to the human being, but according to rule 1, includes research on identifiable human material and identifiable data. Moreover, rule 15 holds that medical research should be conducted only by a scientifically qualified person and under the 
supervision of a clinically competent medical person. The responsibility always rests on the researcher, even if the subject has given consent. The predictable risks or burden of the research have to be carefully assessed in comparison with forseeable benefits to the subject or others. This should be possible in the research on healthy volunteers as well. In rule 16 there is a strange provision: it says, "The design of all studies should be publicly available". Does this mean, that all the studies, their research protocol and their results should be available to others? What is meant by "design"? Are these just the design rules? Research normally is confidential until published and ethical committees are admonished to keep it that way.

Physicians should not do any investigation if the risks outweigh the potential benefits rule 17. This is repeated in rule 18, where it is stated that research involving human subjects should be conducted only if the importance of the objective outweighs the inherent risks and burdens to the subject. If the subject is a healthy volunteer, the research can be of a purely scientific nature. Then follows rule 19, which again is not easy to understand. Medical research is justified only if there is a reasonable likelihood that the populations in which research is carried out stand to benefit from the results of the research. Does this rule out all purely scientific research? If healthy volunteers can take part in research, purely scientific trials should be possible. What is meant by populations? Moreover, is "benefit" more of a general nature? Rule 19 obviously does not have much impact.

There is sloppy wording in rule 20: The subjects must be volunteers and informed participants in the research project. Is this just a repetition of the necessity of informed consent or does the research subject have spontaneously to volunteer to take part in the research? It is easier to follow rule 21 , in respecting the privacy of the subject, the confidentiality of the patient's information and in minimising the impact of the study on the subject's physical and mental integrity.

Rule 22 returns to informed consent. The information has to be very broad. It concerns the aims, methods, sources of funding, any public conflicts of interests, institutional affiliations of the researcher and anticipated benefits and potential risks of the study and discomfort it may entail. After ensuring that the subject has understood the information, the physician should then obtain the subject's freely-given informed consent, preferably in writing. Up to now the information has not always included the sources of funding and the affiliation of the researcher, whatever that means. If the subject is in a dependent relationship to the physician, the physician should be particularly cautious (rule 23). The informed consent should then be obtained by another physician who should be "well-informed". If the research subject is legally incompetent, then the consent has to be given by the legally authorised representative (rule 24). 
Minors and incompetents should not be included in research, unless the research is necessary to promote the health of the population represented and the research cannot be performed on legally competent persons instead. If the minor is able to understand the research, his or her assent is necessary in addition to the consent of the parents (rule 25). If the patient is not able to give consent, including proxy or advanced consent, the research should be done only if the condition that prevents obtaining informed consent is a necessary characteristic of the research population. This should be stated in the experimental protocol for consideration and approval of the review committee. The consent should be obtained as soon as possible from the in dividual or legally authorised surrogate (rule 26).

Ethical committees play a major part in the Declaration of Helsinki. Rule 13 states that the design and performance of each experimental procedure involving human subjects should be clearly formulated in an experimental protocol. This protocol should be submitted for consideration, comment, guidance, and where appropriate, approval to a specially appointed ethical review committee, which must be independent of the investigator, the sponsor and any kind of undue influence. This independent committee should be in conformity with the laws and regulations of the country in which the research experiment is performed. The committee has the right to monitor ongoing trials. The researcher has the obligation to provide information to the committee, especially about any serious adverse events. The researcher should also submit to the committee for review information regarding funding, sponsors, institutions, and affiliations, other potential conflicts of interest and the incentives for subjects.

Rule 13 broadens the task of the ethical committee. The ethical committee is still there for consideration, comment and guidance, but it has to be told about conflicts of interests. If there is any serious adverse event, the ethical committee has to be notified. This places a burden on ethics committees, since all adverse events should be brought to the attention of the committee, even those that happen overseas in multicentre and multinational studies. Some ethical committees have established small sub-committees that deal with that information.

The importance of asking consideration, comment, and guidance and maybe approval is underlined in rule 27. There it is said that reports of experimentation not in accordance with the principles laid down in the Declaration should not be accepted for publication. The publishers of learned journals require the clearance by an ethical committee in accepting papers for publication. That is very real incentive to go before the ethical committee. Moreover, authors and publishers have to preserve the accuracy of the results of the research in publication. Negative as well as positive results should be published or otherwise publicly available. Sources of funding, institutions, affiliations, and any possible conflicts of interest 
should be declared in the publication, a rule that is honoured more in the breach than observance.

The basic principles fall short of some expectations. As far as ethical committees are concerned, one would have liked to hear about the number of members, the fields the members come from, and the scope of consideration by the committee. Moreover, in general, the new Declaration of Helsinki falls short in identifying the modern forms of research. There is mention of proxy or advanced consent and placebo in the rules 26 and 29. One had hoped that the new Declaration of Helsinki would have concerned itself with the specifics of medical research as they are today: controlled clinical studies, double or triple blind studies, crossover, washout, pilot projects, etc. None of them are mentioned in the Declaration.

\section{Medical Research Combined with Medical Care}

If the research is justified by its potential prophylactic, diagnostic, or therapeutic value, the physician may combine it with medical care (rule 28). According to rule 29, the benefits, risks, burdens and effectiveness of a new method should be tested against those of the best current prophylactic, diagnostic, and therapeutic methods. This does not exclude the use of placebo or no-treatment in studies where no proven prophylactic diagnostic or therapeutic method exists and one should add to rule 29 "where there are just minor illnesses or discomforts".

It follows from rule 30 , that at the conclusion of the study every patient entered into the study should be assured of access to the best proven prophylactic, diagnostic and therapeutic methods identified by the study. Here the follow-up treatment of the research subject is asked of the pharmaceutical companies. They have done this mostly in HIV-studies. Now it should become the general rule. Rule 31 repeats the principle of informed consent and states moreover that the refusal of the patient to participate in the study must never interfere with the patient-physician relationship. This obviously means that the patient will get the best treatment available, no matter if he or she agrees to participate in the study or refuses to do so. Rule 32 concerns itself with the hopeless cases. If proven prophylactic, diagnostic, and therapeutic methods do not exist or have been ineffective, the doctor is free to use unproven or new prophylactic, diagnostic and therapeutic measures with the informed consent from the patient if in his or her judgment they offer the hope of saving life, re-establishing health or alleviating suffering.

Where possible, these measures should be made the object of research, designed to evaluate their safety and efficacy. In all cases new information should be recorded and, where 
appropriate, published. This rule allows medical trials in single cases, pilot projects in a number of cases, maybe up to ten or even fifty, and finally clinically controlled studies.

\section{THE NEW RULES OF THE DECLARATION OF HELSINKI}

The Declaration of Helsinki, promulgated in the year 2000 in Edinburgh, Scotland, is a compromise. The conservative attitude of the European countries has mostly prevailed. There is differentiation between therapeutic and non-therapeutic trials. The acceptability of risk is still the main concern for the doctor. Ethical committees have been given more tasks, but in general the Declaration of Helsinki is a paper the researchers and ethical committees can live with.

Where the Declaration of Helsinki remains silent, the local rules take over. This especially is the case as far as responsibility is concerned. In nearly all countries there is liability for negligence. In France, the liability is an objective one if it is purely scientific. In therapeutic trials the responsibility is based on presumed fault. In Germany, pharmaceutical trials are covered by third party insurance. Which of the two approaches, the French or the German one, compensation or insurance, is preferable only time will tell.

\section{THE DECLARATION OF HELSINKI AND THE DIRECTIVES OF THE EUROPEAN UNION}

The European Union is about to issue two directives concerning medical trials - to be more exact, the testing of pharmaceuticals and the clinical trials of medical devices. Both types of trial will be regulated in different directives. Since there is already an understan ding between the European Parliament and the Commission the contents of the directives are known. As far as the testing of pharmaceuticals is concerned, the ethical committees will have very little time - four weeks for the main committee in the case of multicentre studies, and just fourteen days for the local ethics committees. In the case of experimentation on patients who cannot give informed consent, the trial is permitted only if it is of direct benefit to the experimental subject. In this respect the European directive differs from the Declaration of Helsinki, and the Bioethics Convention of the European Union (Strasbourg). There shall be no experimenting on incompetent persons if not for their benefit. Literally, there is the question, whether participating in a clinical controlled study is for the benefit of the experimental subject. Normally the subject has just a $50 \%$ chance of getting the new medication, since the control group is treated according to the normal standard. Take the case of the burn victims that came before the Hanover Ethics Committee: Patients with extensive burns (60\% or more) should undergo a clinically controlled study with a new medication where just $50 \%$ of the research 
population was going to get the new pharmaceutical. The ethics committee finally allowed the research on the ground of presumed consent: The burn victim is not able to give his or her free consent, but it is in his or her best interest to have a chance of being treated in a modern way, since the standard treatment is not very effective. Even a $50 \%$ chance is in the best interest of the patient and will be covered by presumed will.

These directives will become the law of the land, since all the members of the European Union have to adopt these rules. When they have become law, the ethics committees will face a dilemma: In the case of pharmaceutical studies experimentation on incompetents is allowed only if it is for the direct benefit of the research subject. For other studies, even in the testing of new medical devices, experimentation of a purely scientific nature is not prohibited by the Declaration of Helsinki or most local laws and certainly not by the Bioethics Convention, which expressly allows them in section 17 , where trials with minimal risks are permitted. It is hard to understand that the ethics committees will then have to judge by different standards in cases of pharmaceutical trials and all others. The famous words of Genesis 3:5 come to mind: Eritis sicut deus, scientes bonum et malum. 
